\title{
Commerce transfrontalier informel et sécurité alimentaire : le cas du riz en Côte d'Ivoire
}

\author{
Alliou Salihini DIARRASSOUBA ${ }^{1}$, Aubin Nogbou A. AMANZOU², Foungnigué Noé \\ COULIBALY ${ }^{3}$ \\ ${ }^{I}$ UFR Sciences économiques et de gestion, Université Jean Lorougnon Guédé, Daloa, Côte d'ivoire \\ ${ }^{2}$ Unité de recherche Economie des Réseaux, Université Virtuelle de Côte d'ivoire \\ ${ }^{3}$ UFR Sciences économiques et de développement, Université Alassane Ouattara, Bouaké, Côte d'ivoire
}

\begin{abstract}
Résumé : Cet article analyse l'incidence du commerce transfrontalier informel en riz sur la sécurité alimentaire rizicole en Côte d'Ivoire dans le cadre de la politique nationale de vulgarisation de cette denrée. Un modèle de cointégration basé sur l'approche ARDL (Autoregressif à retards échelonnés) a été appliqué. Les résultats montrent l'existence d'une relation à court et à long terme entre le commerce transfrontalier et la sécurité alimentaire en riz. A court terme, une augmentation des flux de détournement frontalier en riz de $10 \%$ entraîne une augmentation de l'insécurité alimentaire en riz en Côte d'Ivoire de $0,2 \%$ alors qu'à long terme, cette augmentation est de $0,17 \%$. Aussi, l'analyse de la causalité avec la méthode de Toda-Yamamoto indique un lien indirect entre le commerce transfrontalier et l'insécurité alimentaire à travers la superficie de riz récoltée. Les pouvoirs publics devraient associer à l'augmentation des superficies cultivables un mécanisme de valorisation des prix bord champs et d'achat anticipé d'une proportion du volume de la production rizicole dans ces zones afin de réduire le commerce transfrontalier informel et renforcer la politique d'autosuffisance dans le secteur.
\end{abstract}

Mots-clés : ARDL, causalité, Côte d'Ivoire, Flux frontaliers, riz, sécurité alimentaire.

\section{Introduction}

La sécurité alimentaire, surtout dans les pays en développement, reste un des enjeux majeurs de la lutte contre la pauvreté et l'amélioration du bien -être des populations. Selon la FAO (2019), 20\% de la population africaine était sous-alimentée en 2017 et 237 millions de personnes en Afrique subsaharienne ont souffert de sous-nutrition chronique en 2019. La région pourrait même compter plus de la moitié des personnes souffrant de faim chronique dans le monde en 2030 si la tendance n'est pas inversée.

Pour faire face à ces enjeux, en Afrique de l'Ouest particulièrement, le riz est un des aliments clés dont la promotion est incluse dans les politiques et programmes visant la sécurité alimentaire afin d'éliminer la faim et la malnutrition d'ici 2030 comme ambitionné par les autorités (FAO,2019). Il représente environ $37 \%$ de la consommation de céréales des ménages ouest-africains ${ }^{1}$.

En Côte d'Ivoire, la sécurité alimentaire repose sur un certain nombre de produits vivriers de base dont le riz reste une composante essentielle selon la Stratégie Nationale de Développement de la riziculture (SNDR, 2020-2030) élaborée par le ministère de la promotion de la riziculture. La

\footnotetext{
${ }^{1}$ Centre européen de gestion des politiques de développement (ECDPM), 2020.
} 
problématique de la sécurité alimentaire à travers cette denrée a induit diverses politiques depuis l'indépendance du pays. D'une approche interventionniste de l'Etat axée sur l'ensemble de la chaîne de valeur à travers l'amélioration de l'approvisionnement en intrants, à la modernisation des techniques culturales, la création des organismes d'assistance technique et la valorisation de la commercialisation. Par exemple, l'on peut citer récemment la création de l'Office National de Développement de la Riziculture en Juillet 2010, la création d'un ministère dédié à la promotion de la culture du riz en 2017, le financement de sept (7) centres de production de semences de qualité à haut rendement couvrant les grandes régions du pays à partir de 2020.

Pourtant la production en riz blanchi n'a évolué que de 1267720 tonnes à 1.3 million de tonnes puis à 1304468 tonnes, de 2012 à 2017 et 2018 (ONDR, 2017 ; SNDR 2020-2030) avec un taux de couverture des besoins de consommation nationale de $85 \%, 50 \%$ et $71 \%$ sur la période. Ce déficit de production en riz face aux besoins nationaux est estimé en moyenne à un million (1.000.000) de tonnes par an, comblé essentiellement par les importations (SNDR, 2020-2030). De plus, la consommation de cette denrée en Côte d'ivoire croît, en moyenne, de $6 \%$ par an.

Cette faiblesse du niveau de production qui fragilise la sécurité alimentaire en riz est expliquée par différents facteurs dans diverses études en Côte d'Ivoire et en Afrique de façon générale : effets des changements climatiques, disponibilité des intrants en quantité et en qualité, les motivations économiques et financières (prix, revenus,...), la législation agricole (Adegnika, 2018 ; Breumier et al, 2018 ; Achy, 2016 ; Ambagna et Foning 2014 ; Lançon,2008).

La solution apportée à ce risque d'insécurité alimentaire en riz est la promotion de sa culture à travers les politiques et programmes mais surtout les importations afin de constituer des stocks de sécurité. Ces importations s'avèrent coûteuses et ont atteint en 2012 plus de 1200000 tonnes et 1 496849 en 2018 de riz blanchi importé (ONDR,2017 ; SNDR, 2020-2030). Soit un coût de plus en plus élevé qui est passé de 200 milliards en 2012 à 385 milliards de FCFA en 2018, impactant négativement la balance des paiements. La dépendance extérieure de la Côte d'Ivoire en matière d'offre de riz blanchi traduit sa vulnérabilité en termes de sécurité alimentaire. Cette vulnérabilité a été perceptible avec la crise économique de 2008 qui a vu certains pays exportateurs (Vietnam, Thaïlande ...) réduire leurs exportations et, récemment en 2020 avec les externalités négatives de la crise liée à la covid-19.

Parallèlement à ces risques, une partie de la production nationale fait l'objet de détournement dans les zones frontalières de grande production depuis quelques années (FAOstats, 2018 ; SNDR, 2018), essentiellement les régions de l'Ouest et du Nord-ouest. Il s'agit d'un commerce transfrontalier entre les riziculteurs ivoiriens desdites régions et la population vivant de l'autre côté de la frontière. Ces régions concernées font d'ailleurs partie des zones ayant bénéficié du financement de centres de production de semences de qualité à haut rendement pour leurs capacités naturelles à booster la production nationale.

Ce commerce informel transfrontalier, certes relatif en volumes il y a quelques années, est en nette progression au cours de cette dernière décennie. Il est passés de 5,11\% de la production totale de riz paddy (105 058 tonnes) en 2014 à près de 10\% de la production nationale (exactement, 8,24\%) en 2018 avec 166207 tonnes (SNDR 2020-2030). Or l'on n'observe pas de mouvements pareils sur le riz en sens inverse susceptibles de compenser ces pertes afin d'évoquer un commerce mutuellement avantageux. Une des explications à ce commerce transfrontalier serait lié à l'avantage d'un meilleur prix recherché par les paysans, surtout que la mise en œuvre des processus de transformation des quantités de riz produites tardent à se concrétiser dans ces régions. En effet, le prix moyen du riz paddy en Côte 
d'ivoire est de 150 FCFA alors que le riz blanchi importé de qualité dite «inférieure » est en moyenne à 500 FCFA (SNDR, 2018).

Ainsi, en sus des facteurs susmentionnés qui fragilisent la sécurité alimentaire, les statistiques de la filière révèlent un nouveau facteur dont l'influence serait susceptible d'aggraver les défis en la matière : les détournements de flux frontaliers de la production de riz paddy dans le cadre de transactions informelles entre les riziculteurs et les populations vivant du côté opposé de la frontière.

La situation du riz en Côte d'Ivoire peut donc être résumée comme suit : la production nationale ne couvre à peine en moyenne qu'entre 60 et $70 \%$ des besoins nationaux, les importations restent coûteuses et fluctuantes selon le contexte économique mondial et une partie des flux nationaux tendent de plus en plus à être détournés vers certains pays frontaliers via un commerce informel.

Dès lors l'intérêt de réexaminer la dynamique des facteurs influençant la production du riz dans le cadre des politiques de promotion de la sécurité alimentaire en la matière en Côte d'Ivoire en y intégrant ce nouveau paramètre. Cela d'autant plus que tous les experts s'accordent sur le fait que l'Asie qui couvre essentiellement la différence des besoins en riz des pays africains sera elle-même importatrice nette de riz à l'horizon 2030 (FAO, 2020). En d'autres termes, quelle est l'influence du commerce transfrontalier informel sur la sécurité alimentaire rizicole en Côte d'Ivoire ? Cette étude entend contribuer, à travers l'analyse de ce facteur, aux politiques et programmes de lutte contre l'insécurité alimentaire en riz, la denrée la plus consommée par la population ivoirienne.

Au niveau théorique, elle s'inscrit dans les approches de la théorie des avantages comparatifs du commerce international et de la modélisation des rendements agricoles qui ont fait l'objet de plusieurs applications dans diverses études dans le domaine en Afrique.

$\mathrm{Au}$ regard de ces constats, ce papier a pour objectif d'analyser l'incidence du commerce transfrontalier informel sur la sécurité alimentaire rizicole en Côte d'Ivoire. De façon spécifique, il cherche à : (i) déterminer la nature de la relation entre le commerce transfrontalier de riz et la sécurité alimentaire rizicole en Côte d'Ivoire ; (ii) mesurer l'influence des échanges de flux de frontières sur la sécurité rizicole dans le cadre des politiques de promotion de l'autosuffisance.

Pour mener l'étude, les hypothèses suivantes sont formulées : (i) l'existence d'une causalité indirecte entre le commerce transfrontalier de riz et la sécurité alimentaire rizicole en Côte d'ivoire ; (ii) les échanges de flux de frontières influencent significativement et négativement la sécurité alimentaire rizicole à court et à long terme en Côte d'ivoire.

Ce papier est structuré en quatre points de la façon suivante : la section 1 présente un aperçu de la littérature sur la problématique. La section 2 aborde la méthodologie à travers la description des données, des variables et la spécification des modèles d'estimation retenus. La section 3 analyse les résultats obtenus.

\section{Aperçu de la littérature}

La question de la sécurité alimentaire s'inscrit dans le cadre général des politiques économiques visant l'amélioration du bien-être des populations dans le secteur agricole. Elle est abordée dans la littérature en termes de recherche de leviers susceptibles d'améliorer le niveau de la production agricole à travers l'analyse des facteurs de production et des questions relatives aux marchés, à la commercialisation. Dès lors, cette étude s'inscrit-elle, au niveau théorique, dans les approches de la 
modélisation des rendements agricoles et des avantages comparatifs liés au commerce international (commerce transfrontalier informel).

Les théories de la modélisation des rendements agricoles essaient d'expliquer le comportement des agriculteurs en cas de changements de prix de la production ou de politiques macroéconomiques influençant le domaine agricole. Nerlove (1956 et 1958), dans «the Nerlovian models of supply response », fut le premier à développer cette théorie. Même si elle fait l'objet de débats, elle reste l'une des plus utilisées dans les études d'estimation de la production agricole relativement aux fluctuations dans le domaine. Nerlove part de deux constats : (i) la réaction des producteurs est fonction du prix actuel sur le marché ; ce qui signifie que la variation du prix d'un produit et celle du stock de production ne sont pas réalisées simultanément, impliquant qu'à court terme l'offre agricole et les prix à la production n'augmentent pas au même rythme ; (ii) la possibilité de mesurer les élasticités de long terme de l'offre du secteur agricole face aux fluctuations des variables l'influençant à partir d'un modèle dynamique d'équations simultanées.

En plus de la théorie de Nerlove, on peut relever la théorie de l'avantage relatif ou comparatif de David Ricardo (1817). Selon son approche, le commerce international reposerait, au-delà de la productivité, sur la rentabilité dégagée par l'échange monétaire entre les acteurs relativement au coût du travail ou à la différence de prix dans le pays de faible productivité. La présente étude repose sur la différence de prix observé par les paysans entre le riz paddy produit en Côte d'Ivoire et le prix proposé dans les pays frontaliers concernés essentiellement du fait de leur faible niveau de production rizicole relative.

Plusieurs études empiriques peuvent être relevées dans le sens sus-évoqué afin de confronter ces théories. Elles portent en général sur les produits vivriers de base des différentes régions qui contribuent à la sécurité alimentaire ou des produits de rente susceptibles d'améliorer les revenus des paysans, accroitre le niveau de vie des populations rurales.

A titre d'illustration, Lançon (2008) en s'appuyant sur une statistique descriptive, a analysé les tendances de l'économie rizicole dans les différentes régions du monde suite à une modification du prix ou de la quantité de production sur les pays exportateurs et importateurs. Pour lui, ces modifications provoquent pour les pays exportateurs, l'accélération de la reconfiguration des flux, avec l'émergence de nouveaux exportateurs et la réorientation des courants commerciaux.

A travers une modélisation économétrique fondée sur l'approche ARDL (autorégressif à retards échelonnés), Ambagna et Foning (2014) montre que la productivité de la terre a un effet plus significatif sur la sécurité alimentaire par rapport à la productivité du travail au Cameroun. Il estime qu'à court terme, une augmentation de $10 \%$ de la productivité du travail engendre un accroissement de $2,5 \%$ des disponibilités alimentaires, tandis que l'accroissement de $10 \%$ de la productivité de la terre accroit de $3,2 \%$ les disponibilités alimentaires. Par ailleurs il souligne qu'à long terme, cet accroissement des disponibilités alimentaires est respectivement de $4,5 \%$ et $6,1 \%$.

Également en Côte d'Ivoire, Achy (2016) considère un modèle de sélection (heckman), dans lequel il analyse l'impact de l'environnement de production sur l'offre de riz domestique. Il trouve une corrélation négative entre l'offre de riz des ménages et l'augmentation du prix de vente du riz à la ferme avec une élasticité de $-0,005$. Ce résultat signifierait que malgré la baisse des prix, les producteurs augmentent leurs productions pour s'assurer un revenu minimum. 
Dans le cadre de la recherche de la sécurité alimentaire en riz au Madagascar, Breumier et al (2018,) à travers une méthodologie centrée sur la méthode IMPRESS ((IMPact des REchercheS au Sud) développée par le CIRAD, ont aussi montré que le riz pluvial d'altitude a une énorme incidence sur l'amélioration de l'autosuffisance et sur le bien-être des ménages d'agriculteurs de la région de Vakinankaratra.

Dans le même sens mais sur la culture de l'igname, Adegnika (2018) en s'appuyant sur un modèle de type nerlovien a analysé les facteurs influençant la production dans la commune de Glazoué au Bénin. Elle montre que le prix de l'igname aux producteurs et la pluviométrie n'ont aucun impact significatif sur cette culture à l'opposé des superficies emblavées et de l'association culturale du coton.

Aussi, Mariam (2018), en s'appuyant sur un modèle de cointégration fondé sur l'approche ARDL (autorégressif à retards échelonnés) cherche à expliquer une relation de long terme entre l'offre de noix de cajou et les facteurs explicatifs de la dynamique de production. A travers ce modèle elle montre que seulement le prix, l'exportation et la superficie affectent positivement la production du cajou à long terme parmi les divers facteurs évoqués.

A l'examen des études susmentionnées, la contribution de cette étude consiste à analyser l'influence spécifique du commerce transfrontalier informel de riz sur la sécurité alimentaire à travers une modélisation économétrique.

\section{Méthodologie}

Cette section décrit les données et les variables ainsi que la spécification du modèle de l'étude.

\subsection{Données de l'étude}

Les données de la présente étude proviennent de la base de données de la FAO, de l'Agence pour le Développement de la filière Riz en Côte d'Ivoire (ADERIZ) et de Google Earth pro.

La production, la superficie récoltée de riz paddy, l'importation de riz blanchi et l'exportation de riz paddy (volume des transactions transfrontalières de riz) ont été collectées à partir des données de la FAO de 1985 à 2018. Quant au prix de la production du riz paddy, il a été collecté à partir des données de l'ADERIZ et de la FAO de 1985 à 2018. Concernant la précipitation, elle provient de Google Earth pro.

Cette étude porte donc sur une série de trente-trois (33) observations; de 1985 à 2018 relativement aux données disponibles.

\subsection{Description des variables}

Les variables ont été constituées à partir de la littérature sur les facteurs de production rizicole et/ ou d'autres aliments dans le cadre de la lutte contre l'insécurité alimentaire et des données disponibles.

Ainsi en référence aux études de Breumier et al (2018), Adegnika (2018), Mariam (2018) et Lançon (2008), l'on peut retenir les variables résumées dans le tableau suivant : 
Tableau 1 : synthèse des variables

\begin{tabular}{|c|c|}
\hline Variables & Notation \\
\hline Sécurité alimentaire : Production du riz paddy (tonnes) & PRO \\
\hline Superficies récoltées & SUP \\
\hline Prix à la production (tonnes de riz) & PRI \\
\hline Importation de riz blanchi & IMP \\
\hline Flux de commerce transfrontalier (Exportation de riz paddy) & PRE \\
\hline Précipitation (mm de pluie) & \\
\hline
\end{tabular}

\section{Source: Auteurs}

\subsection{Modèle économétrique de l'étude : le modèle ARDL}

La méthodologie économétrique adoptée pour examiner le lien entre les flux de détournement de frontière et la sécurité alimentaire rizicole en Côte d'ivoire repose sur une approche ARDL relativement à la littérature (Adegnika, 2018 ; Mariam, 2018 ; Ambagna et Foning ,2014) et la nature des données de l'étude. En effet, compte tenu du nombre réduit d'observations de l'étude (Adegnika, 2018 ; Mariam, 2018), l'analyse de la cointégration dans la présente étude repose sur le modèle ARDL de Pesaran et al. (2001). En plus des propriétés statistiques supérieures pour les échantillons de faible taille, cette approche permet d'analyser la cointégration entre les variables sans se soucier de l'ordre d'intégration des séries. Elle se fonde sur une procédure de tests appelée «bounds tests ». Un modèle à correction d'erreur peut être spécifié à partir du moment où l'hypothèse de cointégration est vérifiée (la valeur $F$ de Fisher est supérieure à la borne critique supérieure). Il faut rappeler que la sécurité alimentaire rizicole est approximée par le niveau de production de riz et les flux de détournement par les exportations de riz dans la présente étude.

Ainsi, la formulation du modèle est établie comme suit :

$$
\begin{aligned}
& \mathrm{LPRO}_{\mathrm{t}}=\alpha_{0}+\sum_{\mathrm{i}=1}^{\mathrm{P}} \beta_{\mathrm{i}} \mathrm{LPRO}_{\mathrm{t}-\mathrm{i}}+\sum_{\mathrm{i}=0}^{\mathrm{q}} \delta_{\mathrm{i}} \mathrm{LEXP}_{\mathrm{t}-\mathrm{i}}+\sum_{\mathrm{i}=0}^{\mathrm{q}} \partial_{\mathrm{i}} \operatorname{LIMP}_{\mathrm{t}-\mathrm{i}}+\sum_{\mathrm{i}=0}^{\mathrm{q}} \mu_{\mathrm{i}} \mathrm{LPRE}_{\mathrm{t}-\mathrm{i}}+ \\
& \sum_{\mathrm{i}=0}^{\mathrm{q}} \mathrm{V}_{\mathrm{i}} \mathrm{LPRI}_{\mathrm{t}-\mathrm{i}}+\sum_{\mathrm{i}=0}^{\mathrm{q}} \gamma_{\mathrm{i}} \mathrm{LSUP}_{\mathrm{t}-\mathrm{i}}+\theta_{1} \mathrm{LPRO}_{\mathrm{t}-1}+\theta_{2} \operatorname{LEXP}_{\mathrm{t}-1}+\theta_{3} \mathrm{LIMP}_{\mathrm{t}-1}+\theta_{4} \mathrm{LPRE}_{\mathrm{t}-1}+ \\
& \theta_{5} \mathrm{LPRI}_{\mathrm{t}-1}+\theta_{6} \mathrm{LSUP}_{\mathrm{t}-1}+\sigma \mathrm{T}+\varepsilon_{\mathrm{t}}
\end{aligned}
$$

De l'équation précédente on a :

$$
\begin{aligned}
& \Delta \text { LPRO }_{\mathrm{t}}=\alpha_{0}+\sum_{\mathrm{i}=1}^{\mathrm{P}} \beta_{\mathrm{i}} \Delta \mathrm{LPRO}_{\mathrm{t}-\mathrm{i}}+\sum_{\mathrm{i}=0}^{\mathrm{q}} \delta_{\mathrm{i}} \Delta \mathrm{LEXP}_{\mathrm{t}-\mathrm{i}}+\sum_{\mathrm{i}=0}^{\mathrm{q}} \partial_{\mathrm{i}} \Delta \mathrm{LIMP}_{\mathrm{t}-\mathrm{i}}+\sum_{\mathrm{i}=0}^{\mathrm{q}} \mu_{\mathrm{i}} \Delta \mathrm{LPRE}_{\mathrm{t}-\mathrm{i}} \\
& +\sum_{\mathrm{i}=0}^{\mathrm{q}} \mathrm{v}_{\mathrm{i}} \Delta \mathrm{LPRI}_{\mathrm{t}-\mathrm{i}}+\sum_{\mathrm{i}=0}^{\mathrm{q}} \gamma_{\mathrm{i}} \Delta \mathrm{LSUP}_{\mathrm{t}-\mathrm{i}}+\theta_{1} \mathrm{LPRO}_{\mathrm{t}-1}+\theta_{2} \mathrm{LEXP}_{\mathrm{t}-1}+\theta_{3} \mathrm{LIMP}_{\mathrm{t}-1} \\
& +\theta_{4} \mathrm{LPRE}_{\mathrm{t}-1}+\theta_{5} \mathrm{LPRI}_{\mathrm{t}-1} \\
& +\theta_{6} \text { LSUP }_{\mathrm{t}-1}+\sigma \mathrm{T}+\varepsilon_{\mathrm{t}}
\end{aligned}
$$

$\mathrm{LPRO}_{\mathrm{t}}$ : Production du riz paddy au temps t et en tonnes (en logarithmes : $\mathrm{L}$ )

LEXP : Exportation du riz paddy

LIMP : Importation du riz blanchi 
LPRE : La précipitation

LPRIX : Le prix à la production

LSUP : La superficie récoltée

$\mathrm{T}$ : le trend ; l'introduction d'un trend dans l'équation de cointégration permet de capter l'influence du temps sur la variable dépendante et l'effet des variables non spécifiées dans le modèle comme le développement des infrastructures rizicoles, la gouvernance dans le domaine, les différentes reformes et le fonctionnement du marché (Adegnika, 2018).

\section{Analyse des résultats}

Cette section présente et analyse les résultats des tests préliminaires au modèle ARDL ainsi que les résultats de la nature des liens. Mais avant elle aborde les statistiques descriptives des données de l'étude.

\subsection{Statistiques descriptives}

Certaines particularités peuvent être relevées à partir de l'analyse des variables de la présente étude.

Tableau 2 : Synthèse des statistiques descriptive des variables

\begin{tabular}{ccccccc}
\hline Variables & EXP & PRO & IMP & PRE & PRI & SUP \\
\hline Moyenne & 7659,29 & 947319,60 & 570972,50 & 98,22 & 128604,00 & 517981,00 \\
Médiane & 3762,50 & 679075,00 & 524028,50 & 98,48 & 120247,00 & 493894,50 \\
Maximum & 38918,00 & 2153000,00 & 1384155,00 & 129,03 & 287000,00 & 833672,00 \\
Minimum & 0,00 & 540000,00 & 253008,00 & 78,78 & 60000,00 & 339939,00 \\
\hline
\end{tabular}

Source : auteurs

L'on relève que la production moyenne en riz en Côte d'ivoire est estimée à 947319,6 tonnes sur la période 1985 - 2018 alors que les flux de commerce de frontières sont évalués en moyenne à 7659,29 tonnes de riz (tableau 2). De plus la production varie de 540.000 tonnes à 2109333 tonnes de 1985 à 2018 tandis que le commerce transfrontalier atteint parfois presque les 40.000 tonnes sur la période (Graphiques 1 et 2). Ces résultats montrent que la production nationale reste faible associée à une part de plus en plus croissante de déperditions de ladite production du fait du commerce frontalier.

Graphique 1 : évolution de la production de riz paddy de 1985 à 2018 en Côte d'Ivoire

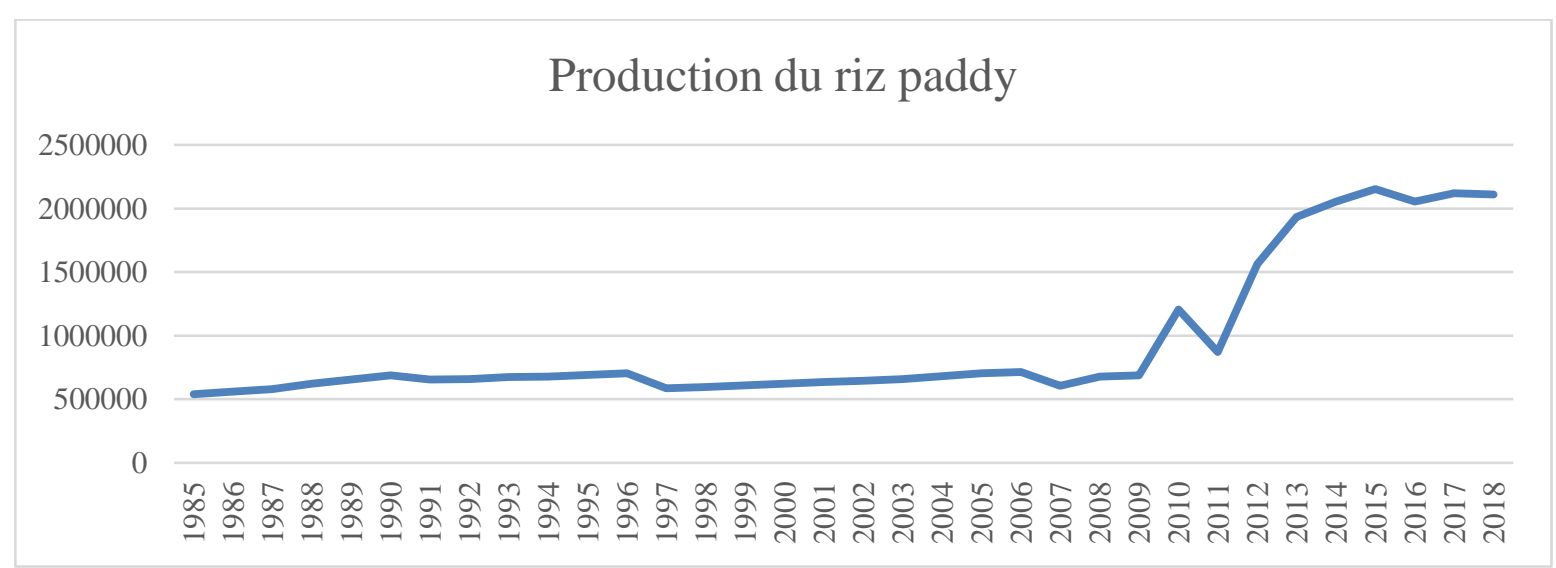

Source : auteurs 
Graphique 2 : évolution des flux du commerce transfrontalier de riz de 1985 à 2018 en Côte d'Ivoire

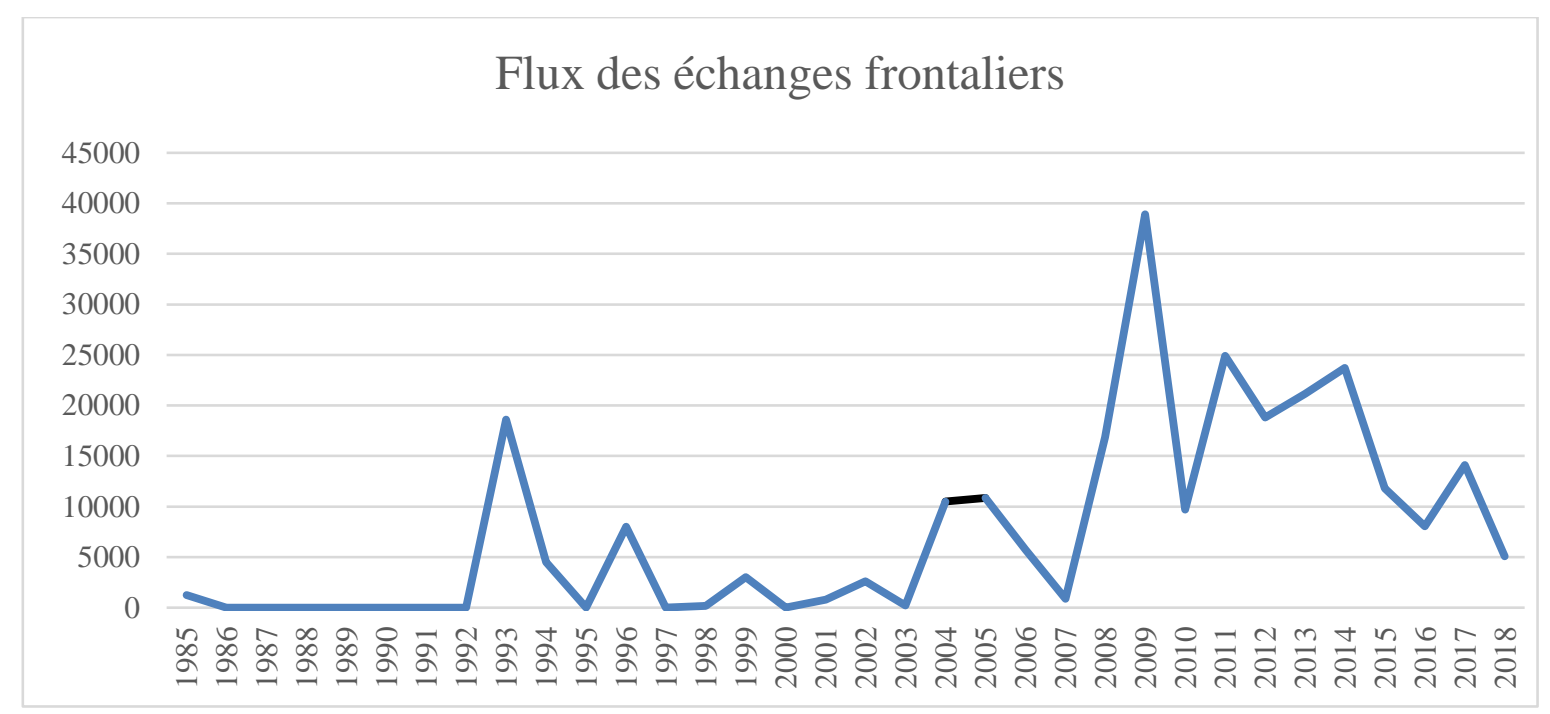

Source : auteurs

Parallèlement, le volume des importations de riz blanchi afin de faire face aux besoins nationaux, représente, en moyenne, plus de la moitié de la production nationale. Ces importations sont en moyenne de 570972,50 tonnes sur la période (tableau 2) afin de faire face aux besoins nationaux de consommation.

Ces résultats révèlent la nécessité, dans le cadre de la lutte contre l'insécurité alimentaire en riz, d'une analyse approfondie des résultats de la modélisation économétrique de l'incidence de ces flux détournés sur le stock de production du riz en Côte d'Ivoire.

\subsection{Résultats de tests préliminaires : racine unitaire, cointégration aux bornes}

\subsubsection{Test de racine unitaire}

Selon Charemza and Deadman (1992), la plupart des analyses macroéconomiques sur des séries temporelles subissent des perturbations d'origine diverses qui tendent à modifier la variance des données qui biaise parfois les résultats des estimations. D'où la nécessité d'analyser au préalable la stationnarité des séries et y apporter des corrections ci-nécessaire.

Les résultats indiquent que les variables intégrées d'ordres I(0) et I(1). Ce résultat permet de confirmer l'application du modèle ARDL.

En effet, dans cette étude, partir du test de racine unitaire de Dickey-Fuller Augmented (ADF), l'on vérifie s'il y a présence de racine unitaire (série non stationnaire) ou pas (série stationnaire).

Le tableau 3 (ci-dessous) montre que les variables du modèle qui sont stationnaires en niveau (leurs valeurs d'ADF supérieurs aux valeurs critiques) sont : la précipitation ; l'importation du riz blanchi et l'exportation du riz paddy.

Par contre le tableau 4 (ci-dessous) indique que les variables prix à la production, superficie récoltée et production du paddy qui n'étaient pas stationnaires en niveau sont stationnaires en différence première (valeurs d'ADF sont supérieures aux valeurs critiques). 
Tableau 3 : Résultat de test de stationnarité en niveau

\begin{tabular}{|l|l|l|l|}
\hline Variables & ADF T-Statistique & Valeur critique de seuil de 5\% & Stationnarité \\
\hline PRO & $-1,157146$ & $-3,552973$ & Non \\
\hline EXP & $-4,276664$ & $-3,552973$ & Oui \\
\hline IMP & $-3,678784$ & $-3,557759$ & Oui \\
\hline PRE & $-5,370156$ & $-3,552973$ & Oui \\
\hline PRI & $-2,613028$ & $-3,552973$ & Non \\
\hline SUP & $-1,044987$ & $-3,552973$ & Non \\
\hline
\end{tabular}

Source : auteurs

Tableau 4 : Résultats de test de stationnarité en différence première

\begin{tabular}{|l|l|l|l|}
\hline Variables & ADF T-Statistique & $\begin{array}{l}\text { Valeur critique de } \\
\text { seuil de 5\% }\end{array}$ & Stationnarité \\
\hline PRI & $-6,323276$ & $-3,557759$ & Oui \\
\hline PRO & $-7,030953$ & $-3,557759$ & Oui \\
\hline SUP & $-5,082673$ & $-3,557759$ & Oui \\
\hline
\end{tabular}

Source : auteurs

\subsubsection{Test de cointégration de Pesaran et al. (2001) ou cointégration aux bornes ou bounds test to cointegration}

Pour tester l'existence de la relation de court ou de long entre des variables économiques, la littérature économétrique fournit plusieurs tests ou approches. Les variables de l'étude étant intégrées d'ordres différents (I (0), I (1)) et à Mariam (2018), Adegnika (2018), l'on peut recourir au test de cointégration de Pesaran et al. (2001) pour vérifier l'existence d'une ou plusieurs relations de cointégration entre les variables.

Ce test a été développé au départ par Pesaran et Shin (1999) et est qualifié de l'approche ARDL (approach to cointegrating) ou test de cointégration aux bornes. Il nécessite deux étapes : (i)déterminer le décalage optimal (AIC, SIC) ; (ii) recourir au test de Fisher pour tester la cointégration entre séries.

Pour déterminer le décalage optimal, le critère d'information de Schwarz (SIC) est utilisé (Chaque lag de ARDL se rapporte à une variable précise suivant leur classement dans l'output). Selon le graphique 3 (ci-dessous), sur les vingt meilleurs modèles, selon le critère d'information Schwarz, le modèle qui offre la plus petite valeur SIC est le modèle $\operatorname{ARDL}(1,4,3,2,4,4)$. Il est donc considéré comme le modèle ARDL optimal dans cette étude. 
Graphique 3 : Valeurs graphiques SIC

Schwarz Criteria (top 20 models)

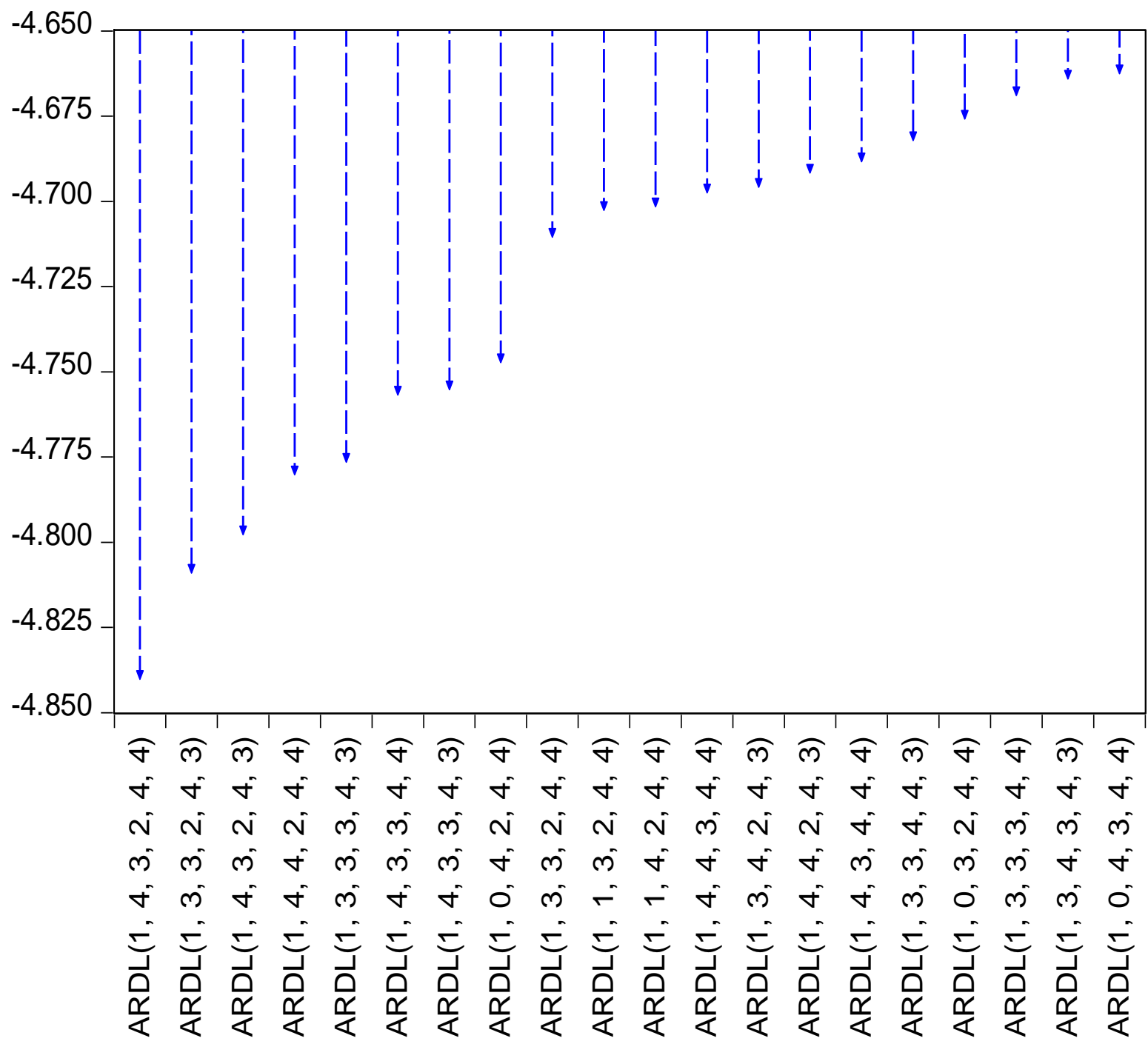

Source : auteurs

Dans cette étude, les résultats du test de cointégration aux bornes confirment l'existence d'une relation de cointégration entre les différentes variables (Tableau 5 ci-dessous) car la valeur F de Fisher de la statistique est largement supérieure à celle de la borne critique supérieure pour les différents seuils de significativité $(1 \% ; 2,5 \% ; 5 \%$ et $10 \%)$.

Tableau 5 : résultat des bounds test

\begin{tabular}{lccc}
\hline Seuil critique & Borne critique inferieure & Borne critique supérieure & Valeur de la statistique \\
\hline $1 \%$ & 3,5 & 4,63 & \\
$2,50 \%$ & 3,11 & 4,13 & 24,49232 \\
$5 \%$ & 2,81 & 3,76 & \\
$10 \%$ & 2,49 & 3,38 & \\
\hline
\end{tabular}

Source : auteurs 
Ainsi, les résultats de la dynamique de court terme en lien avec ceux de long terme sont obtenus à partir d'un modèle à correction d'erreur suivant l'approche ARDL. Selon les estimations, il existe une relation de court terme et de long terme, de façon globale, entre les variables de l'étude. Plus spécifiquement, ces résultats révèlent un lien de court terme et de long terme entre les flux de commerce transfrontalier et la sécurité alimentaire en riz (Tableau 6 ci-dessous) avec un lien de long terme plus faible en valeur (Tableau 7 ci-dessous).

En effet, le coefficient d'ajustement ou force de rappel (CointEq (-1)) est statistiquement significatif (probabilité $=0,000$ ) et négatif ; ce qui garantit un mécanisme de correction d'erreur, et donc l'existence d'une relation de long terme (cointégration) entre variables.

De façon générale, le tableau 6 montre que la sécurité alimentaire est significativement et négativement influencée par le niveau de production du riz de l'année antérieure à court terme. L'on relève qu'elle est significativement influencée par l'ensemble des variables explicatives mais différemment.

Quant aux flux de commerce de frontières, ils influencent significativement et positivement le niveau de la production de l'année $\mathrm{t}$ à court terme. Une augmentation de $1 \%$ des exportations induit un accroissement de 0,02\% du niveau de la production en année t.

Tableau 6 : Modèle à correction d'erreur suivant l'approche ARDL (Elasticités de court terme)

\begin{tabular}{lcccc}
\hline Variable & Coefficient & Ecart-type & t-Statistic & Prob, \\
\hline LPRO(-1) & $-0,893264$ & 0,157518 & $-5,670859$ & 0,0024 \\
D(LEXP) & 0,019575 & 0,001865 & 8,889841 & 0,0003 \\
D(LEXP(-1)) & 0,007916 & 0,002417 & 3,27564 & 0,0221 \\
D(LEXP(-2)) & 0,00855 & 0,002378 & 3,595915 & 0,0156 \\
D(LEXP(-3)) & 0,004372 & 0,001904 & 2,296413 & 0,0701 \\
D(LIMP) & $-0,171102$ & 0,019409 & $-8,815723$ & 0,0003 \\
D(LIMP(-1)) & 0,296808 & 0,021462 & 13,829678 & 0 \\
D(LIMP(-2)) & 0,263022 & 0,031249 & 8,416917 & 0,0004 \\
D(LPRE) & 0,484249 & 0,03707 & 13,063173 & 0 \\
D(LPRE(-1)) & $-0,527008$ & 0,052303 & $-10,076095$ & 0,0002 \\
D(LPRI) & $-0,240029$ & 0,034176 & $-7,023275$ & 0,0009 \\
D(LPRI(-1)) & $-0,618572$ & 0,038247 & $-16,173212$ & 0 \\
D(LPRI(-2)) & $-0,717377$ & 0,051975 & $-13,802406$ & 0 \\
D(LPRI(-3)) & $-0,332003$ & 0,039622 & $-8,379338$ & 0,0004 \\
D(LSUP) & 0,643525 & 0,051853 & 12,410559 & 0,0001 \\
D(LSUP(-1)) & $-0,541443$ & 0,057997 & $-9,335761$ & 0,0002 \\
D(LSUP(-2)) & $-0,370361$ & 0,064143 & $-5,774018$ & 0,0022 \\
D(LSUP(-3)) & 0,120469 & 0,054712 & 2,201896 & 0,0789 \\
C & $-2,421843$ & 0,125539 & $-19,291491$ & 0 \\
@ TREND & 0,016771 & 0,001407 & 11,923543 & 0,0001 \\
CointEq(-1) & $-1,893264$ & 0,097485 & $-19,421167$ & 0,0000 \\
\hline
\end{tabular}

Source : auteurs

Ce résultat suggèrerait une corrélation négative entre le commerce transfrontalier informel et la sécurité alimentaire à court terme. En d'autres termes, une augmentation des flux de commerce de frontières de riz de $1 \%$, toutes choses égales par ailleurs, induit une augmentation de l'insécurité alimentaire en riz en Côte d'Ivoire à court terme de $0,02 \%$.

Ce résultat est conforme à ceux de Mariam (2018) qui trouvent que les exportations de noix de cajou ont un effet positif sur la production à court terme et ceux de Lançon (2008) qui trouve qu'une 
modification des exportations de riz induit un ajustement du niveau de production du riz dans les pays importateurs de riz.

Il pourrait s'expliquer par le fait que les producteurs de riz cherchent le prix le plus intéressant entre les deux frontières et se déplacent de la frontière ivoirienne vers le pays le plus offrant : le Libéria, la Guinée, le Ghana. Toute chose qui influence négativement le niveau de la production nationale effectivement vendue sur le territoire national et pourrait exacerber l'insécurité alimentaire.

Toutefois, les effets de ce phénomène restent atténués dans le temps car la relation de long terme entre les deux variables est certes significative et positive, mais relativement plus faible en valeur selon les résultats de l'estimation dans le tableau 7 ci-dessous. Une augmentation des flux de détournements de frontières de riz de $1 \%$, toutes choses égales par ailleurs, induit une augmentation de l'insécurité alimentaire en riz en Côte d'Ivoire à long terme de $0,017 \%$. Ce résultat de long terme est conforme à ceux de Mariam (2018) même s'il faut relever que ladite étude porte sur l'offre de noix de cajou.

Tableau 7 : Elasticités de long terme

\begin{tabular}{lcccc}
\hline Variable & Coefficient & Ecart-type & t-Statistic & Prob, \\
\hline LEXP & 0,01743 & 0,044445 & 5,310962 & 0.0093 \\
LIMP & $-0,239984$ & 0,045268 & $-5,301442$ & 0,0032 \\
LPRE & 0,980359 & 0,081902 & 11,969919 & 0,0001 \\
LPRI & 0,093731 & 0,034928 & 2,683526 & 0,0436 \\
LSUP & 1,028849 & 0,021688 & 47,437669 & 0 \\
@ TREND & 0,016771 & 0,001407 & 11,923543 & 0,0001 \\
\hline
\end{tabular}

Source : auteurs

\subsection{Analyse de la Causalité de Granger au sens de Toda-Yamamoto}

L'objectif du test de causalité est de déterminer l'origine de la variation d'une variable par rapport à une autre à long terme. C'est-à-dire voir si la première variable cause la seconde variable ou si la seconde variable cause la première ou encore s'il existe une relation causale bidirectionnelle entre les deux variables au-delà de la simple corrélation positive ou négative entre elles comme analysée par la cointégration. Ceci, en vue d'améliorer l'efficacité prévisionnelle des modèles économétriques utilisés. L'on recourt traditionnellement au test de causalité de Granger. Mais quand l'on a des variables non stationnaires qui ne sont pas cointégrées ou sont intégrées à des ordres différents, le test de causalité de Granger s'avère inefficace. Pour pallier, l'on recourt au test de causalité au sens de Toda-Yamamoto (1995) qui est basé sur la statistique « W » de Wald, distribuée suivant un khi-deux. L'hypothèse nulle implique alors l'absence de causalité entre variables (probabilité $\chi^{2}>5 \%$ ). Tels sont les arguments qui justifient ce choix d'évaluation de la causalité entre les variables de façon générale et spécifiquement entre les variables d'intérêt dans la présente étude.

L'analyse des résultats de la causalité au sens de Toda-Yamamoto entre les flux de commerce transfrontalier et l'insécurité alimentaire en riz (Tableau 8 ; ci-dessous) montre une absence de causalité directe entre ces deux variables. Cependant l'on relève une causalité indirecte entre les deux allant des flux de commerce transfrontalier vers l'insécurité alimentaire à travers la superficie récoltée. 
Tableau 8 : Résultats Tests de Causalité de Toda-Yamamoto

\begin{tabular}{lcccccc}
\hline Variables & \multicolumn{7}{c}{ Variables explicatives ou causales } \\
\cline { 2 - 7 } dépendantes & LPROD & LEXP & LIMP & LPRE & LPRI & LSUP \\
\hline LPROD & - & 1,7885 & 1,2295 & 0,5565 & $6,2629^{* *}$ & $8,4586^{* *}$ \\
& & $(0.4089)$ & $(0,5408)$ & $(0,7571)$ & $(0,0437)$ & $(0,0146)$ \\
LEXP & 1,5076 & - & 2,3745 & 2,3431 & 1,5764 & 0,8597 \\
& $(0,4706)$ & & $(0,3051)$ & $(0,3099)$ & $(0,4547)$ & $(0,6506)$ \\
LIMP & $9,7885^{* * *}$ & 0,0379 & - & 4,3236 & 1,1326 & 3,1809 \\
& $(0,0075)$ & $(0,9812)$ & & $(0,1151)$ & $(0,5676)$ & $(0,2038)$ \\
LPRE & 0,1907 & 1,8764 & 2,3815 & - & 0,0153 & 0,5251 \\
& $(0,9090)$ & $(0,3913)$ & $(0,3040)$ & & $(0,9924)$ & $(0,7691)$ \\
LPRI & $5,0236^{*}$ & $5,3320^{*}$ & 0,2736 & 0,0740 & - & 0,5003 \\
& $(0,0811)$ & $(0,0695)$ & $(0,8722)$ & $(0,9637)$ & & $(0,7787)$ \\
LSUP & $4,6764 *$ & $7,0434 * *$ & 0,1471 & 3,8762 & 0,4036 & - \\
& $(0,0965)$ & $(0,0295)$ & $(0,9291)$ & $(0,1440)$ & $(0,8172)$ & \\
\hline
\end{tabular}

Source : auteurs ; probabilité entre parenthèse $(\ldots) .{ }^{*}$ : significatif à $10 \% ; * *$ : significatif à $5 \%$. ${ }^{* *}$ : significatif à $1 \%$

En effet, les estimations montrent une relation de causalité allant du commerce transfrontalier à la superficie récoltée $(0,0295<5 \%)$, et de la superficie récoltée à l'insécurité alimentaire $(0,0146<5 \%)$, Le commerce transfrontalier informel de riz n'influe pas directement sur l'insécurité alimentaire; il passe par les superficies récoltées pour expliquer indirectement le niveau d'insécurité alimentaire en riz en Côte d'Ivoire.

En d'autres termes, les résultats de cette étude montrent que les superficies de riz récoltées sont causées par l'anticipation des flux de commerce transfrontalier de riz et que le niveau desdites superficies pourrait induire une fragilisation de la sécurité alimentaire en riz en Côte d'Ivoire.

\section{Conclusion}

Cette étude avait pour objectif d'analyser l'incidence du commerce transfrontalier informel sur la sécurité alimentaire rizicole en Côte d'Ivoire.

A travers une approche ARDL, les résultats de la cointégration aboutissent à l'existence d'un lien de court et de long terme entre les flux de commerce transfrontalier informel et l'insécurité alimentaire rizicole. De plus, les estimations de la causalité au sens de Toda-Yamamoto montrent une relation de causalité indirecte allant desdits flux vers l'insécurité alimentaire par l'intermédiaire des superficies de surface récoltées de riz.

Ainsi dans le cadre des politiques et programmes d'autosuffisance en riz afin de mieux planifier les ambitions futures de pays exportateur de riz, les autorités pourraient accentuer un certain nombre de mesures. Entre autres : renforcer l'aménagement et le rendement des surfaces rizicoles cultivées (récoltées) ; l'augmentation des superficies récoltées par les riziculteurs devrait être accompagnée d'un mécanisme de valorisation des prix bord champs tenant compte des prix proposés par les pays voisins afin d'éviter les détournements de flux ; les actions visant à améliorer la productivité des riziculteurs devraient être accompagnés de mécanismes d'achat anticipé d'une proportion du volume de leur production probable ; les actions visant à faciliter l'écoulement de la production rizicole à l'intérieur du pays devraient être renforcées. 


\section{BIBLIOGRAPHIE}

[1] FAO, Organisation des Nations Unies pour l'Alimentation et l'Agriculture, "Aperçu régional de la sécurité alimentaire et de la nutrition en Afrique”, http://www.fao.org/news/story/fr/item/1062875/icode/_2019.

[2] Ministère de la promotion de la riziculture, "Stratégie Nationale de Développement de la Riziculture (SNDR 2020 2030)”, 2020.

[3] ONDR , Office National de Développement de la Riziculture, "Statistique rizicole", http:// www.ondr.ci/statistique_production.php, 2017

[4] M. Adegnika, "Estimation de type Nerlovien des déterminants de la production de l'igname dans la commune de Glazoue au Benin", Revue Marocaine de Gestion et d'Economie, 2018, Vol 4, No8, pp.36-57, http://revues.imist.ma/?journal=RMGE.

[5] P. Breumier, A. Ramarosandratana, A. Ramanantsoanirina, K. Vom Brocke, C. Marquié, M-H. Dabat, et L-M. Raboin, "Évaluer les impacts des recherches en agriculture sur la société : outils, méthodes, études de cas", Cah. Agric,2018, Vol.27, N¹, pp.271-300. https://doi.org/10.1051/cagri/2017065

[6] L. Achy, "Analyse économétrique de l'offre du riz local en Côte d'Ivoire", International Journal of Innovation and Applied Studies,2016, Vol. 17, Nº1, pp.291-314. journals.org/links/papers.php/IJIAS-16-093-01

http://www.issr-

[7] J. Ambagna et M. Foning, "Productivité des facteurs de production agricole et disponibilités alimentaires au Cameroun. Conférence: 8es Journées de Recherches en Sciences Sociales", https://www.researchgate.net/publication/271440980, 2014.

[8] F. Lançon, “Le rôle des échanges internationaux du riz dans la régulation de l'économie mondiale du riz”, Document de travail CIRAD, https://agritrop.cirad.fr/556656/1/document_556656.pdf, 2008.

[9] M. Nerlove, "Estimates of the Elasticities of Supply of Selected of Agricultural Commodities", Journal of Farm Economics, 1956, Vol. 38, N², pp. 496-509

[10] M. Nerlove, The Dynamics of Supply: Estimation of farmers responses to price. Johns Hopkins University Press, Baltimore, 1958, pp.268.

[11] M. Nerlove, "Distributed Lags and Unobserved Components in Economics Time Series in Ten Economic Studies", In the Tradition of irving Fisher, W. Fellner, ed., New York John Wiley \& Sons, pp. 127-169, 1967.

[12] M. Nerlove, "The Dynamics of Supply: Retrospect and Prospect", American Journal of Agricultural Economics Studies, 1979, N 61, pp. 874-888.

[13] Von J-H. Thünen, “Der Isolierte Staat ln Beziehung auf Landwirtschaft und Nationalöconomie ”, Vol. I, 1826, Hamburg, Perthes. Traduction française par Laverrière J. ,1851, Recherches sur l'influence que le prix des grains la richesse du sol et les impôts exercent sur les systèmes de culture. Paris, Guillaumin. Vol. II, Section 1, 1850, Rostock, Leopold. Traduction française par Volkoff M., 1857, Le salaire naturel et son rapport au taux de 1'intérêt. Paris, Guillaumin. Vol. II, Section 2 et Vol. III, éd. posthume par Schumacher H . ,1863, Rostock, Hinstorff.

[14] D. Ricardo, On the Principles of Political Economy and Taxation, John Murray, London,1817, In: Sraffa, P., Ed., The Works and Correspondence of David Ricardo, Vol. 1, Cambridge University Press, Cambridge, 1951.

[15] O. G. Mariam, “Analyse de la dynamique de l'offre de noix brutes de cajou en Côte d'Ivoire : une application par l'approche autorégressif à retards échelonnés (ARDL) ”, Eur. Sci. J, 2018, Vol.14, N³4, pp.292-306., http://dx.doi.org/10.19044/esj.2018.v14n34p292

[16] M. H. Pesaran, Y. Shin, et R.J. Smith, "Bounds Testing Approaches to the Analysis of Level Relationships", Journal of Applied Econometrics, 2001, Vol.16, pp.289-236.

[17] W. Charemza and D. Deadman, "New Directions in Econometric Practice: General to Specific Modelling, Cointegration, and Vector Autoregression", Journal of applied econometrics, 1992, Vol.9, N¹, pp. 99-101. https://doi.org/10.1002/jae.3950090108

[18] D.A. Dickey et W. A. Fuller, "Distribution of the estimtors for autoregressive time series with a unit root", Journal of the American Statistical Association, 1979, Vol.74, N³66, pp. 427-431.

[19] C. W. J. Granger, "Testing for Causality: A Personal Viewpoint”, Journal of Economic Dynamics and Control, 1980, Vol.2, pp. 329-352 (http://dx.doi.org/10.1016/0165-1889(80)90069-X).

[20] C. W. J. Granger, "Causality, cointegration and control”, Journal of Economic Dynamics and Control, 1988, Vol.12, $\mathrm{N}^{\circ}$ 2-3, pp. 551-559.

[21] H.Y. Toda and T. Yamamoto, "Statistical Inference in Vector Autoregressions with Possibly Integrated Processe", Journal of Econometrics, 1995, Vol. 66, pp. 225-250 (http://dx.doi.org/10.1016/0304-4076(94)01616-8). 